\title{
Stability of delayed neural networks with impulsive strength-dependent average impulsive intervals
}

\author{
Huan Zhang ${ }^{\mathrm{a}}$, Wenbing Zhang ${ }^{\mathrm{a}, *}$, Zhi Li ${ }^{\mathrm{b}}$ \\ a Department of Mathematics, YangZhou University, Jiangsu, 225002, China. \\ ${ }^{b}$ Business School of SiChuan University, Chengdu, SiChuan, 610044, China.
}

Communicated by M. Bohner

\begin{abstract}
This paper mainly deals with the stability of delayed neural networks with time-varying impulses, in which both stabilizing and destabilizing impulses are considered. By means of the comparison principle, the average impulsive interval and the Lyapunov function approach, sufficient conditions are obtained to ensure that the considered impulsive delayed neural network is exponentially stable. Different from existing results on stability of impulsive systems with average impulsive approach, it is assumed that impulsive strengths of stabilizing and destabilizing impulses take values from two finite states, and a new definition of impulsive strength-dependent average impulsive interval is proposed to characterize the impulsive sequence. The characteristics of the proposed impulsive strength-dependent average impulsive interval is that each impulsive strength has its own average impulsive interval and therefore the proposed impulsive strength-dependent average impulsive interval is more applicable than the average impulsive interval. Simulation examples are given to show the validity and potential advantages of the developed results.
\end{abstract}

Keywords: Neural networks, impulsive strength-dependent average impulsive interval, time-varying impulse, stability.

2010 MSC: 34K20, 34K45.

(C)2018 All rights reserved.

\section{Introduction}

Over the past few years, neural networks have been widely studied due to their potential applications in various fields, such as, robotic control, fault detection, system identification, image restoration and so on $[2,10,13,18,24,25,29-32,36]$. In the implementation of neural networks, owing to the limited speed of signal propagation, time-varying delays are often encountered. The resulting neural networks with various time delays were extensively studied, and many significant results have been obtained [7, $14,17,20,21,35,40,43]$, see [7, 14, 43] for discrete time delay, [21] for distributed time delay and [17] for reaction-diffusion delay.

\footnotetext{
*Corresponding author

Email address: zwb850506@126.com (Wenbing Zhang)
}

doi: $10.22436 /$ jnsa.011.05.02

Received: 2017-11-07 Revised: 2018-01-29 Accepted: 2018-03-01 
On the other hand, due to switching phenomenon, frequency change, or other sudden noise, impulsive effects often appear in real systems [1, 9, 12, 19, 28, 34, 37]. Generally, impulses can affect the dynamical behaviors of the networks, if a network is not stable and the impulsive effects are beneficial to stability, then the network can achieve stability with an appropriate impulsive interval [23, 38]. Conversely, if a network is stable and the impulsive effects are harmful to stability, then the impulses may destroy stability [35]. Hence, it is necessary to investigate its influences on the stability of neural networks. According to the influence of impulses on stability, impulses can be classified into the following two categories: 1) an impulsive sequence is said to be stabilizing if the impulsive effects can enhance the stability of dynamical systems; 2) an impulsive sequence is said to be destabilizing if it can suppress the stability of the dynamical system. Over the past few years, many well-studied results have been obtained on stability of neural networks with stabilizing or destabilizing impulses. For example, in [23], by means of average impulsive interval, an unified criterion for the synchronization of impulsive complex networks including synchronizing and desynchronizing impulses was obtained. In [39], exponentially synchronization problem was investigated for a class of coupled switched neural networks with mode-dependent impulsive effects, where desynchronizing and synchronizing impulsive effects were considered, respectively. Besides, in many practical systems such as electronic systems or biological systems, the stabilizing and destabilizing impulses can exist simultaneously, so it is of great significance to investigate the stability of the systems with both stabilizing and destabilizing impulses. In [38], upper and lower bounds were used, respectively for destabilizing and stabilizing impulses to characterize impulsive sequence, some sufficient conditions were obtained to guarantee the stability of the delayed neural networks with time-varying impulses. As is shown in [23], compared with the lower and upper bounds, the average impulsive interval approach can obtain less conservative results since average impulsive interval can be applied to a wider range. Thus, the results obtained in [38] may be conservative to some extent, and it is interesting to investigate the stability problem of delayed neural networks with both stabilizing and destabilizing impulses by using the average impulsive interval approach. Although, the average impulsive interval approach has been applied to many existing literatures, there are no results concerning the time-varying impulses, where both stabilizing and destabilizing impulses are considered simultaneously. In addition, in most existing results on impulsive systems with average impulsive approach [4, 15, 16, 35], it is assumed that the average impulsive interval is independent of the impulsive strength (we call it as strength-independent average impulsive interval), and the average impulsive intervals for all impulsive sequences are assumed to be equal to each other, which may not be anticipated. Actually, when considering time-varying impulsive effects, there are many impulses with different impulsive strengths. Thus, the strength-independent average impulsive interval approach may be conservative, since the average impulsive interval is computed by strength-independent parameters. It is straightforward that such an assumption for all impulsive sequences in a strength-independent manner may results in conservativeness. Inspired by the ideas of mode-dependent averaged well time in switched systems [42], in this paper, a new definition of impulsive strength-dependent average impulsive interval is proposed to reduce the conservativeness in strength-independent average impulsive interval approach.

In this paper, we aim to investigate the stability of delayed neural networks with time-varying impulses, where both stabilizing and destabilizing impulses are considered simultaneously. A new definition of strength-dependent average impulsive interval is proposed to characterize the impulsive sequences, where each impulsive strength has its own average impulsive interval. Then, with the help of the Lyapunov function approach and the comparison principle, sufficient conditions have been obtained to ensure the stability of the neural networks under consideration. Finally, numerical simulations are given to verify the effeteness and advantages of our theoretical findings.

Notations: In this paper, $\mathbb{R}^{n}$ denotes the $n$-dimensional Euclidean space. $\mathbb{N}_{+}$denotes the set of positive integers. The superscript "T" represents the matrix or vector transposition. For $x \in \mathbb{R}^{n},|x|$ denotes the Euclidean norm of $x$. For matrix $A \in \mathbb{R}^{n \times n},\|A\|=\sqrt{\lambda_{\max }\left(A^{\top} A\right)}$, where $\lambda_{\max }(\cdot)$ represents the largest eigenvalue. $\operatorname{diag}\{\ldots\}$ stands for a block-diagonal matrix. The identity matrix of dimension $n$ is devoted by $I_{n}$. For $\psi: \mathbb{R} \rightarrow \mathbb{R}$, the Dini derivative of $\psi(t)$ is defined as $D^{+} \psi(t)=\overline{\lim }_{s \rightarrow 0^{+}}(\psi(t+s)-$ 
$\psi(t)) /$ s. Given $\tau>0, C\left([-\tau, 0], \mathbb{R}^{\mathfrak{n}}\right)$ denotes the family of continuous functions from $[-\tau, 0]$ to $\mathbb{R}^{\mathfrak{n}}$.

\section{Model formulation and preliminaries}

The model formulation and some preliminaries will be presented in this section. The following neural networks model will be considered,

$$
\dot{x}(t)=A x(t)+B h(x(t))+\operatorname{Ch}(x(t-\tau(t)))+I,
$$

where $x(t)=\left[x_{1}(t), x_{2}(t) \ldots, x_{n}(t)\right]^{\top}$ is the state vector associated with the neurons. $A=\operatorname{diag}\left\{a_{1}, a_{2} \ldots\right.$, $\left.a_{n}\right\}<0$ is the self feedback matrix; $B=\left\{\left(b_{i j}\right)\right\}_{\mathfrak{n} \times \mathfrak{n}}$ and $D=\left\{\left(d_{i j}\right)\right\}_{\mathfrak{n} \times \mathfrak{n}}$ are the connection weighted matrices; $h(x(t))=\left(h_{1}\left(x(t), h_{2}\left(x(t), \ldots, h_{n}(x(t))\right)^{T}\right.\right.$ denotes the activation functions of the neurons, $I \in$ $\mathbb{R}^{n}$ is constant external input, $\tau(t)$ is the time-varying delay satisfying $0<\tau(t)<\tau$. The nonlinear function $h(\cdot)$ satisfies the following assumption.

Assumption 2.1. The nonlinear function $\mathrm{h}(\cdot)$ satisfies global Lipschitz condition, i.e., there exists a positive definite diagonal matrix $\mathrm{L}=\operatorname{diag}\left\{\mathrm{l}_{1}, \mathrm{l}_{2}, \ldots, \mathrm{l}_{\mathrm{n}}\right\}$ such that for any $\mathrm{x}_{1}, \mathrm{x}_{2} \in \mathbb{R}^{\mathrm{n}}$, the following condition holds:

$$
\left|h\left(x_{1}\right)-h\left(x_{2}\right)\right| \leqslant L\left|x_{1}-x_{2}\right| .
$$

Let $x^{*}$ be the equilibrium point of (2.1). For the purpose of simplicity, we can shift the intended equilibrium $x^{*}$ to be original by letting $u=x-x^{*}$, and then the system in (2.1) can be transformed into

$$
\dot{u}(t)=A u(t)+B f(u(t))+C f(u(t-\tau(t)),
$$

where $u(t)=\left[u_{1}(t), u_{2}(t) \ldots, u_{n}(t)\right]^{\top} \in \mathbb{R}^{n}$ is the state vector of the transformed system. It follows from (2.2) that the function $f(u)=h\left(u+x^{*}\right)-h\left(x^{*}\right)$ satisfies

$$
|f(x)-f(y)| \leqslant L|x-y|
$$

for any $x, y \in \mathbb{R}^{n}$. By considering impulsive effects, we have the following impulsive model:

$$
\begin{cases}\dot{u}(t)=A u(t)+B f(u(t))+C f(u(t-\tau(t))), & t \neq t_{k} \\ u\left(t_{k}^{+}\right)=\alpha_{k} u\left(t_{k}^{-}\right), & t=t_{k}\end{cases}
$$

where $\left\{t_{1}, t_{2}, t_{3}, \ldots\right\}$ is a sequence of strictly increasing impulsive moments, $\alpha_{k} \in \mathbb{R}$ represents the strength of impulses at $t_{k}$. We assume that $u(t)$ is right continuous at $t=t_{k}$, i.e., $u\left(t_{k}\right)=u\left(t_{k}^{+}\right)$. Hence, the solutions of (2.3) are piecewise right-hand continuous functions with discontinuities at $t=t_{k}$ for $k \in \mathbb{N}_{+}$. Without loss of generality, we assume that $t_{0}=0$ and the initial condition of (2.3) is given by $x(\mathrm{t})=\phi(\mathrm{t}) \in \mathrm{C}\left([-\tau, 0], \mathbb{R}^{\mathrm{n}}\right)$.

Remark 2.2. It can be easily obtained from the second equation of (2.3) that if the impulsive strength $\left|\alpha_{k}\right|>1$, the impulses are destabilizing and when the impulsive strength $\left|\alpha_{k}\right|<1$, the impulses are stabilizing. In this paper, both destabilizing and stabilizing impulses are considered simultaneously. In what follows, we assume that the impulsive strengths of destabilizing impulses take values from a finite set $\left\{\mu_{1}, \mu_{2} \ldots, \mu_{N}\right\}$ and the impulsive strengths of stabilizing impulses take values from a finite set $\left\{v_{1}, v_{2} \cdots, v_{M}\right\}$, where $\left|\mu_{i}\right|>1,\left|v_{j}\right|<1$, for $i=1,2, \cdots, N, j=1,2, \cdots, M$.

Devote by $N_{i}$ and $\bar{N}_{j}$, respectively the number of destabilizing impulses with impulsive strength $\mu_{i}$ and the number of stabilizing impulses with impulsive strength $v_{j}$.

Assumption 2.3. The average impulsive interval of the destabilizing impulses with impulsive strength $\mu_{\mathrm{i}}$ is not less than $\varepsilon_{i}$ and the average impulsive interval of the stabilizing impulses with impulsive strength $v_{j}$ is not more than $\epsilon_{j}$, if there exist positive numbers $\varepsilon_{i}$ and $\epsilon_{j}, i=1,2, \ldots, M, j=1,2, \ldots, M$ such that

$$
N_{i} \leqslant \frac{t-s}{\varepsilon_{i}}+N_{0}, N_{j} \geqslant \frac{t-s}{\epsilon_{j}}-\bar{N}_{0}
$$

where $\mathrm{N}_{0}, \overline{\mathrm{N}}_{0}$ are two positive integers. 
The following definition and lemmas will be used later on.

Remark 2.4. Assumption 2.3 constructs a novel set of impulsive signals with strength-dependent average impulsive interval. It can be seen from Assumption 2.3 that each impulsive strength has its own average impulsive interval in this paper. Compared with the traditional impulsive sequences with average impulsive interval, where all the impulsive strengths have the same average impulsive interval. Our impulsive sequence can be applied to a wider range since the strength-dependent average impulsive interval can provide more profound characterization on the impulsive sequence. Simulation results will show this point in detail.

Definition 2.5. The neural networks in (2.3) are said to be exponentially stable if there exist positive constants $M_{0}>0, \lambda>0$, and $T_{0}>0$ such that for any initial values,

$$
|u(t)|^{2} \leqslant M_{0} e^{-\lambda t}
$$

hold for all $t \geqslant T_{0}$.

Lemma 2.6. For any vectors $x, y \in \mathbb{R}^{n}$, we have $2 x^{\top} y \leqslant x^{\top} x+y^{\top} y$.

Lemma 2.7. Let $0 \leqslant \tau_{i}(t) \leqslant \tau, F\left(t, x, \bar{x}_{1}, \bar{x}_{2}, \ldots, \bar{x}_{m}\right): \mathbb{R}^{+} \times \mathbb{R} \times \ldots \times \mathbb{R} \rightarrow \mathbb{R}$ be nondecreasing in $\bar{u}_{i}$ for each fixed $\left(t, x, \bar{x}_{1}, \bar{x}_{2}, \ldots, \bar{x}_{i-1}, \bar{x}_{i+1}, \ldots, \bar{x}_{m}\right), i=1,2, \ldots, m$, and $\mathrm{I}_{k}(x): \mathbb{R} \rightarrow \mathbb{R}$ be nondecreasing $i n x$. Suppose that

$$
\left\{\begin{array}{l}
D^{+} x(t) \leqslant F\left(t, x(t), x\left(t-\tau_{1}(t)\right), \ldots, x\left(t-\tau_{m}(t)\right)\right) \\
x\left(t_{k}{ }^{+}\right) \leqslant I_{k} x\left(t_{k}{ }^{-}\right), k \in \mathbb{N}_{+}
\end{array}\right.
$$

and

$$
\left\{\begin{array}{l}
D^{+} y(t)>F\left(t, y(t), y\left(t-\tau_{1}(t)\right), \ldots, y\left(t-\tau_{m}(t)\right)\right) \\
y\left(t_{k}^{+}\right) \geqslant I_{k} y\left(t_{k}{ }^{-}\right), k \in \mathbb{N}_{+}
\end{array}\right.
$$

Then $\mathrm{x}(\mathrm{t}) \leqslant \mathrm{y}(\mathrm{t})$ for $-\tau \leqslant \mathrm{t} \leqslant 0$ implies that $\mathrm{x}(\mathrm{t}) \leqslant \mathrm{y}(\mathrm{t})$ for $\mathrm{t} \geqslant 0$.

\section{Main results}

In this section, the exponentially stability problem for the impulsive neural network in (2.3) will be investigated by means of the comparison principle and the Lyapunov function method.

Theorem 3.1. Consider the neural networks (2.3) with strength-dependent average impulsive interval. Suppose that Assumptions 2.1 and 2.3 hold. Then, the neural networks in (2.3) with strength-dependent average impulsive interval will be globally exponentially stable if the following inequality holds

$$
\alpha-\mathrm{Rq}>0,
$$

where $\alpha=-\left(p+\sum_{i=1}^{N} \frac{2 \ln \left|\mu_{i}\right|}{\varepsilon_{i}}+\sum_{j=1}^{M} \frac{2 \ln \left|v_{j}\right|}{\epsilon_{j}}\right), p=\lambda_{\max }\left(2 A+B^{\top} B+L^{\top} L+C^{\top} C\right), q=\lambda_{\max }\left(L^{\top} L\right)$.

Proof. Let $X(t)=u^{\top}(t) u(t)$. Then the derivative of $X(t)$ along the trajectories of the system (2.3) can be obtained as follows:

$$
D^{+} X(t)=u^{\top}(t)\left(A+A^{\top}\right) u(t)+2 u^{\top}(t) B f(u(t))+2 u^{\top}(t) C f(u(t-\tau(t))), t \in\left[t_{k-1}, t_{k}\right), k \in \mathbb{N}_{+} .
$$

By Lemma 2.6, we can get

$$
\begin{gathered}
2 u^{\top}(t) B f(u(t)) \leqslant u^{\top}(t) B^{\top} B u(t)+f^{\top}(u(t)) f(u(t)), \\
2 u^{\top}(t) C f(u(t-\tau(t))) \leqslant u^{\top}(t) C^{\top} C u(t)+f^{\top}(u(t-\tau(t))) f(u(t-\tau(t))) .
\end{gathered}
$$


From Assumption 2.1, we have

$$
\begin{gathered}
f^{\top}(u(t)) f(u(t)) \leqslant u^{\top}(t) L^{\top} L u(t), \\
f^{\top}(u(t-\tau(t))) f\left(u(t-\tau(t)) \leqslant u^{\top}(t-\tau(t)) L^{\top} L u(t-\tau(t)) .\right.
\end{gathered}
$$

From (2.4)-(3.6), we can get

$$
\begin{aligned}
D^{+} X(t) & \leqslant u^{\top}(t)\left(2 A+B^{\top} B+L^{\top} L+C^{\top} C\right) u(t)+u^{\top}(t-\tau(t)) L^{\top} L u(t-\tau(t)) \\
& \leqslant \lambda_{\max }\left(2 A+B^{\top} B+L^{\top} L+C^{\top} C\right) X(t)+\lambda_{\max }\left(L^{\top} L\right) X(t-\tau(t)) \\
& =p X(t)+q X(t-\tau(t)), t \in\left(t_{k-1}, t_{k}\right], k \in \mathbb{N}_{+} .
\end{aligned}
$$

For $t=t_{k}$, from the second equation of (2.3), we get

$$
X\left(t_{k}{ }^{+}\right)=\alpha_{k}^{2} X\left(t_{k}{ }^{-}\right) .
$$

For any $\varepsilon>0$, let $y(t)$ be a unique solution of the following impulsive delay system

$$
\begin{cases}\dot{y}(t)=p y(t)+q y(t-\tau(t))+\varepsilon, & t \neq t_{k}, \\ y\left(t_{k}\right)=\alpha_{k}^{2} y\left(t_{k}-\right), & t=t_{k}, k \in \mathbb{N}_{+}, \\ y(s)=|\phi(s)|^{2}, & -\tau \leqslant t \leqslant 0\end{cases}
$$

Note that $X(s) \leqslant\|\phi(s)\|^{2}=y(s)$ for $-\tau \leqslant t \leqslant 0$, it follows from (3.7), (3.8), and Lemma 2.7 that

$$
y(t) \geqslant X(t) \geqslant 0, t \geqslant 0
$$

By the formula for the variation of parameters, it follows from (3.9) that

$$
y(t)=W(t, 0) y(0)+\int_{0}^{t} W(t, s)[q y(s-\tau(s))+\varepsilon] d s,
$$

where $W(t, s), t, s \geqslant 0$ is the Cauchy matrix of linear system

$$
\begin{cases}\dot{y}(t)=p y(t), & t \neq t_{k}, \\ y\left(t_{k}\right)=\alpha_{k}^{2} y\left(t_{k}^{-}\right), & t=t_{k}, k \in \mathbb{N}_{+} .\end{cases}
$$

According to the representation of the Cauchy matrix, one can obtain the following estimation:

$$
W(t, s)=e^{p(t-s)} \prod_{s<t_{k} \leqslant t} \alpha_{k}^{2} .
$$

For any $t>0$, if there exists an $s(0<s<t)$ such that there are $N_{i}$ (with the impulsive strength $\mu_{i}$ ) destabilizing impulses and $\bar{N}_{j}$ (with the impulsive strength $v_{j}$ ) stabilizing impulses in the interval $(s, t)$, then from Assumption 2.3, we can easily get $N_{i} \leqslant \frac{t-s}{\varepsilon_{i}}+N_{0}, \bar{N}_{j} \geqslant \frac{t-s}{\epsilon_{j}}-\bar{N}_{0}$. Hence, it follows from Assumption 2.3 and (3.12) that

$$
\begin{aligned}
W(t, s) & \leqslant e^{p(t-s)} \prod_{i=1}^{N} \prod_{j=1}^{M}\left|\mu_{i}\right|^{2 N_{i}}\left|v_{j}\right|^{2 \bar{N}_{j}} \\
& \leqslant e^{p(t-s)} \prod_{i=1}^{N} \prod_{j=1}^{M}\left|\mu_{i}\right|^{2\left(\frac{t-s}{\varepsilon_{i}}+N_{0}\right)}\left|v_{j}\right|^{2\left(\frac{t-s}{\varepsilon_{j}}-\bar{N}_{0}\right)} \\
& =e^{p(t-s)} \prod_{i=1}^{N} \prod_{j=1}^{M}\left|\mu_{i}\right|^{2 N_{0}}\left|v_{j}\right|^{-2 \bar{N}_{0}}\left(e^{\ln \left|\mu_{i}\right|}\right)^{2 \frac{t-s}{\varepsilon_{i}}}\left(e^{\ln \left|v_{j}\right|}\right)^{2 \frac{t-s}{\varepsilon_{j}}}
\end{aligned}
$$




$$
\begin{aligned}
& =\prod_{i=1}^{N} \prod_{j=1}^{M}\left|\frac{\mu_{i}^{2 N_{0}}}{v_{j}^{2 \bar{N}_{0}}}\right| e^{p(t-s)} e^{\left(2 \frac{\ln \left|\mu_{i}\right|}{\varepsilon_{i}}+2 \frac{\ln \left|v_{j}\right|}{\epsilon_{j}}\right)(t-s)} \\
& =\prod_{i=1}^{N} \prod_{j=1}^{M}\left|\frac{\mu_{i}^{2 N_{0}}}{v_{j}^{2 \bar{N}_{0}}}\right| e^{p(t-s)} \cdot e^{\frac{2 \ln \left|\mu_{i}\right|}{\varepsilon_{i}}(t-s)} \cdot e^{\frac{2 \ln \left|v_{j}\right|}{\epsilon_{j}}(t-s)} \\
& =\prod_{i=1}^{N} \prod_{j=1}^{M}\left|\frac{\mu_{i}^{2 N_{0}}}{v_{j}^{2 \bar{N}_{0}}}\right| e^{\left(p+\frac{2 \ln \left|\mu_{i}\right|}{\varepsilon_{i}}+\frac{2 \ln \left|v_{j}\right|}{\epsilon_{j}}\right)(t-s)}=R e^{-\alpha(t-s)},
\end{aligned}
$$

where $R=\left|\frac{\mu_{i}^{2 N_{0}}}{v_{j}^{2 N_{0}}}\right|$. Let $\eta=R \sup _{-\tau \leqslant s \leqslant 0}|\phi(s)|^{2}$. Furthermore, it can be derived from (3.11) and (3.13) that

$$
y(t) \leqslant \eta e^{-\alpha t}+\int_{0}^{t} \operatorname{Re} e^{-\alpha(t-s)}[q y(s-\tau(s))+\varepsilon] d s .
$$

Define

$$
h(\theta)=\theta-\alpha+\operatorname{Rq}^{\theta \tau} .
$$

It follows from (3.1) that $h(0)<0$. Since $h(+\infty)=+\infty$ and $h^{\prime}(\theta)>0$, there exists a unique $\lambda$ such that

$$
\lambda-\alpha+\operatorname{Rq}^{\lambda \tau}=0 .
$$

On the other hand, it is obvious from (3.1) that $R^{-1} \alpha-q>0$. Hence,

$$
y(t)=|\phi(t)|^{2} \leqslant \eta \leqslant \eta e^{-\lambda t}+\frac{\varepsilon}{R^{-1} \alpha-q},-\tau \leqslant t \leqslant 0 .
$$

Then we will show that

$$
y(t)<\eta e^{-\lambda t}+\frac{\varepsilon}{R^{-1} \alpha-q} .
$$

If the inequality (3.16) is not true, there exists a $t^{*}>0$ such that

$$
y\left(t^{*}\right) \geqslant \eta e^{-\lambda t^{*}}+\frac{\varepsilon}{R^{-1} \alpha-q}
$$

and

$$
y(t)<\eta e^{-\lambda t}+\frac{\varepsilon}{R^{-1} \alpha-q}, t<t^{*} .
$$

From (3.14) and (3.18), one has

$$
\begin{aligned}
\mathrm{y}\left(\mathrm{t}^{*}\right) & \leqslant \eta e^{-\alpha \mathrm{t}^{*}}+\int_{0}^{\mathrm{t}^{*}} \operatorname{Re}^{-\alpha\left(\mathrm{t}^{*}-\mathrm{s}\right)}[\mathrm{qy}(\mathrm{s}-\tau(\mathrm{s}))+\varepsilon] \mathrm{ds} \\
& <\mathrm{e}^{-\alpha \mathrm{t}^{*}}\left\{\eta+\frac{\varepsilon}{\mathrm{R}^{-1} \alpha-\mathrm{q}}+\int_{0}^{\mathrm{t}^{*}} \operatorname{Re}^{\alpha s}\left[\mathrm{q}\left(\eta \mathrm{e}^{-\lambda(\mathrm{s}-\tau(\mathrm{s}))}+\frac{\varepsilon}{\mathrm{R}^{-1} \alpha-\mathrm{q}}\right)+\varepsilon\right] \mathrm{d} s\right\} .
\end{aligned}
$$

It is derived from (3.15) and (3.19) that

$$
y\left(t^{*}\right)<e^{-\alpha t^{*}}\left\{\eta+\frac{\varepsilon}{R^{-1} \alpha-q}+R q \eta e^{\lambda \tau} \int_{0}^{t^{*}} e^{(\alpha-\lambda) s} d s+\int_{0}^{t^{*}}\left(\frac{q \varepsilon}{R^{-1} \alpha-q}+\varepsilon\right) R e^{\alpha s} d s\right\}
$$




$$
\begin{aligned}
& =e^{-\alpha t^{*}}\left\{\eta+\frac{\varepsilon}{R^{-1} \alpha-q}+R q \eta e^{\lambda \tau} \int_{0}^{t^{*}} e^{(\alpha-\lambda) s} d s+\int_{0}^{t^{*}}\left(\frac{\alpha \varepsilon}{R^{-1} \alpha-q}\right) e^{\alpha s} d s\right\} \\
& =e^{-\alpha t^{*}}\left\{\eta+\frac{\varepsilon}{R^{-1} \alpha-q}+\eta(\alpha-\lambda)\left[\frac{e^{(\alpha-\lambda) t^{*}}}{\alpha-\lambda}-\frac{1}{\alpha-\lambda}\right]+\frac{\alpha \varepsilon}{R^{-1} \alpha-q}\left[\frac{e^{\alpha t^{*}}}{\alpha}-\frac{1}{\alpha}\right]\right\} \\
& =e^{-\alpha t^{*}}\left\{\eta+\frac{\varepsilon}{R^{-1} \alpha-q}+\eta\left(e^{(\alpha-\lambda) t^{*}}-1\right)+\frac{\varepsilon e^{\alpha t^{*}}}{R^{-1} \alpha-q}-\frac{\varepsilon}{R^{-1} \alpha-q}\right\} \\
& =e^{-\alpha t^{*}}\left\{\eta+\frac{\varepsilon}{R^{-1} \alpha-q}+\eta e^{(\alpha-\lambda) t^{*}}-\eta+\frac{\varepsilon}{R^{-1} \alpha-q} e^{\alpha t^{*}}-\frac{\varepsilon}{R^{-1} \alpha-q}\right\} \\
& =\eta e^{-\lambda t^{*}}+\frac{\varepsilon}{R^{-1} \alpha-q},
\end{aligned}
$$

which contradicts to (3.17), so (3.16) holds. Letting $\varepsilon \rightarrow 0$, one can get from (3.10) that

$$
X(t) \leqslant y(t) \leqslant \eta e^{-\lambda t} .
$$

By definition 2.1, the solution $u(t)$ of the impulsive neural networks (2.3) is exponentially stable. This completes the proof of Theorem 2.1.

Remark 3.2. The method of maximum eigenvalue has been used to characterize the impulsive strength in paper, which is conservative to some extent. In the following part, we discuss the case that the impulsive strength is replaced by a full-matrix as what have been studied in literatures $[3,5,6]$. We just consider the condition of $t=t_{k}$ here, since that the situations of $\left(t_{k}, t_{k+1}\right]$ are very similar. Consider the following system:

$$
\begin{cases}\dot{u}(t)=A u(t)+B f(u(t))+C f(u(t-\tau(t))), & t \neq t_{k} \\ u\left(t_{k}^{+}\right)=J u\left(t_{k}^{-}\right), & t=t_{k} .\end{cases}
$$

1. When the Lyapunov function is chosen as $V(t)=x^{\top}(t) x(t)$, we can use

$$
\left(\begin{array}{cc}
-\mu \mathrm{I} & \mathrm{J}^{\mathrm{T}} \\
\mathrm{J} & -\mathrm{I}
\end{array}\right)<0
$$

to replace the maximum eigenvalue approach, which can reduce the conservatism.

2. To be more general, let the Lyapunov function be $V(t)=x^{\top}(t) P x(t)$, we can use

$$
\left(\begin{array}{cc}
-\mu \mathrm{I} & \mathrm{J}^{\mathrm{T}} \mathrm{P} \\
\mathrm{PJ} & -\mathrm{P}
\end{array}\right)<0
$$

to replace the maximum eigenvalue approach, where I is the identity matrix.

3. There are also other ways to reduce the conservatism, such as using time-dependent Lyapunov function [41]. For more details, please refer to [41].

Remark 3.3. In [38], stability problem was investigated for delayed neural networks with time-varying impulses, where both stabilizing and destabilizing impulses were considered simultaneously. However, in [38], lower and upper bounds were used to characterize the impulsive sequence. Clearly, the average impulsive interval approach can provide wider applications than the lower and upper bounds since high-density impulses are allowed to happen in a certain interval with the compensation of low-density impulses [35]. In addition, in [35], it is assumed that all the impulsive strengths have the same average impulsive interval. In order to compare our results with [35], we assume that there are two impulsive sequences, the impulsive interval of the first one is $\epsilon_{1}$ and the impulsive interval of the second one is $\epsilon_{2}$, if we use the average impulsive interval approach derived in [35], one may obtain that both $\epsilon_{1}$ and $\epsilon_{2}$ are greater than a constant $T_{a}$. However, using our strength-dependent average impulsive interval approach, one can obtain that $\epsilon_{1} \geqslant T_{a, 1}$ and $\epsilon_{2} \geqslant T_{a, 2}$. Clearly, our approach is less conservative than the result in [35], which can therefore be applied to wider range. Simulations will show this point in detail. 
Remark 3.4. In [27], a systematic way was proposed to design the event-triggered rules to stabilize a class of linear reset control systems. Then based on Lyapunov-based techniques, stability was investigated for sampled-data systems. In [11], the asymptotic stability of impulsive delay dynamical systems (IDDS) was investigated by using the Lyapunov-Krasovskii method and looped-functionals. Compared with these results, the contributions of this paper can be summarized as the following two aspects. Firstly, the system in [11] is linear system, while the system in this paper is nonlinear system, which is more extensive. Secondly, the time delay $h$ in [11] is a constant, while the time delay $\tau(t)$ in this paper is time-varying, which changes with time. To be more specify, in this paper, if we let $B=0$ and $\tau(t)=h$ in the model (2.3), then the model in this paper becomes the model in [11]. Therefore, the model in this paper is more general than that in [11].

Remark 3.5. The problem of exponential stability was studied for a class of time-delayed systems with impulsive effects in [8] and the main tool is the construction of an impulse-time-dependent complete Lyapunov functional. By comparing with [8], we can get the following differences. Firstly, the time delay $\tau$ is a constant, while the time delay in this paper is time-varying. Moreover, in this paper, a new definition of impulsive-strength dependent average impulsive approach is used to characterize the impulsive sequence, which is more general and less conservative. Very recently, it was shown in [11, 33] that by using the looped Lyapunov-Krasovskii functionals, one can get less conservative results. However, as the main purpose of this paper is to propose a new definition of impulsive strength-dependent average impulsive interval, we don't handle this problem here and we intend to do some further researches based on the methods derived in these papers in the near future.

Remark 3.6. In this paper, the time delay $\tau(t)$ considered is assumed to be bounded and the bound $\tau$ plays an important role in deriving the main results of this paper. Recently, literatures $[22,26]$ proposed the definition of quenching and explained that quenching occurs when stability (instability) of a time-delay system with fixed time-delays within a certain interval is lost when the delay is assumed to be timevarying inside the same interval and vice-versa, which is very interesting. The literature [26] aimed to propose the new concept of quenching, which is indeed valuable. Nevertheless, as the main purpose of this paper is to propose a new definition of strength-dependent average impulsive interval to characterize impulsive sequences, we intend to do some further researches based on the methods derived in [26] in the near future.

The following two Corollaries show that our results can be extended into the stability of neural networks with stabilizing and destabilizing impulses, respectively.

Corollary 3.7. Consider the neural networks in (2.3) with stabilizing time-varying impulses. Suppose that Assumptions 2.1 and 2.3 hold. Then the neural networks in (2.3) will be globally exponentially stable if the following inequality holds

$$
\bar{\alpha}-\overline{\mathrm{R}} q>0,
$$

where $\bar{\alpha}=-\left(p+\sum_{j=1}^{M} \frac{2 \ln \left|v_{j}\right|}{\epsilon_{j}}\right), \bar{R}=\prod_{j=1}^{M}\left|\frac{1}{v_{j}}\right|^{N_{0}}, 0<\left|v_{j}\right|<1$, and the other parameters have been defined in Theorem 2.1.

Corollary 3.8. Consider the neural networks (2.3) with destabilizing time-varying impulses. Suppose that Assumptions 2.1 and 2.3 hold. Then, the neural networks in (2.3) will be globally exponentially stable if the following inequality holds

$$
\tilde{\alpha}-\tilde{R} q>0,
$$

where $\tilde{\alpha}=-\left(p+\sum_{i=1}^{N} \frac{2 \ln \left|\mu_{i}\right|}{\varepsilon_{i}}\right), \tilde{R}=\prod_{i=1}^{N}\left|\mu_{i}\right| N_{0},\left|\mu_{i}\right|>1$, and the other parameters have been defined in Theorem 2.1.

Moreover, if we do not consider time delay in (2.3), then the following corollary can be obtained directly. 
Corollary 3.9. Consider the neural networks in (2.3) without time delay. Suppose that Assumptions 2.1 and 2.3 hold. Then the neural networks in (2.3) will be globally exponentially stable if the following inequality holds

$$
\alpha>0,
$$

where $\alpha=-\left(p+\sum_{i=1}^{N} \frac{2 \ln \left|\mu_{i}\right|}{\varepsilon_{i}}+\sum_{j=1}^{M} \frac{2 \ln \left|v_{j}\right|}{\epsilon_{j}}\right), p=\lambda_{\max }\left(2 A+B^{\top} B+L^{\top} L\right)$.

\section{Numerical simulations}

Example 4.1. Consider the neural networks in (2.3) with stabilizing time-varying impulses, where

$$
\begin{aligned}
A & =-\left(\begin{array}{cc}
1.3 & 0 \\
0 & 1.3
\end{array}\right), B=\left(\begin{array}{ll}
1.3 & 0.1 \\
0.2 & 0.2
\end{array}\right), C=\left(\begin{array}{cc}
0.4 & 0.2 \\
0 & 0.2
\end{array}\right), \\
f(u(t)) & =\tanh (0.8 u(t)), \tau(t)=0.5 \sin (t)+0.5 .
\end{aligned}
$$

After calculating, we can simply get $p=-0.0352, q=0.64$. Assume that there are two impulsive sequences, one is the impulsive sequence with the impulsive strength $v_{1}=0.9$ and average impulsive interval $\epsilon_{1}=0.5$, the impulsive strength of the other one is $v_{2}=0.6$. Using Corollary 3.8, the impulsive neural network in (2.3) is stable if $\epsilon_{2}<3.87$. If we use the lower and upper bounds derived in [38] with $\sup _{k \in \mathbb{N}^{+}}\left\{t_{2 k \downarrow}-t_{2(k-1) \downarrow}\right\}=0.7339$. Let $\epsilon_{2}=2.53$, we can get $\tilde{\alpha}-\tilde{R} q<0$ by using the results in [38]. Hence, it is unable to judge the stability of the impulsive neural networks with $\sup _{k \in \mathbb{N}^{+}}\left\{t_{2 k \downarrow}-t_{2(k-1) \downarrow}\right\}=0.7339$. Consequently, our results are less conservative than the ones in [38]. Moreover, the comparison with [23] is listed in Table 1. Clearly, if we use the method derived in [23], one cannot get the average impulsive interval $\varepsilon_{2}$ since it is assumed that all the impulses have a common average impulsive interval. This means that our impulsive strength-dependent average impulsive approach is more general than the traditional average impulsive interval approach.

Table 1: Maximal allowable average impulsive interval.

\begin{tabular}{|c|c|}
\hline Method & Data comparison \\
\hline Theorem 1 in [23] & $\tau_{\mathrm{a}}=2.1038, \mu=1.2$ \\
\hline Theorem 2.1 & $\varepsilon_{1}=2.1038, \mu_{1}=1.2$ \\
\hline & $\varepsilon_{2}=1.4842, \mu_{2}=1.4$ \\
\hline & $\varepsilon_{3}=1.7330, \mu_{3}=1.6$ \\
\hline
\end{tabular}

\section{Conclusion}

In this paper, stability of delayed neural networks with strength-dependent average impulsive interval has been investigated. First of all, a new definition of strength-dependent average impulsive interval is proposed to characterize the impulsive strengths, where each impulsive strength has its own average impulsive interval. By means of the comparison principle and the Lyapunov function method, sufficient conditions have been obtained to ensure that the neural network under consideration is exponentially stable. Finally, simulations have been presented to show the effectiveness and less conservativeness of our results.

\section{Acknowledgment}

This work was supported in part by the National Natural Science Foundation of China under Grant 61503328. 


\section{References}

[1] D. Antunes, J. Hespanha, C. Silvestre, Stability of networked control systems with asynchronous renewal links: an impulsive systems approach, Automatica J. IFAC, 49 (2013), 402-413. 1

[2] S. Arik, Stability analysis of delayed neural networks, IEEE Trans. Circuits Systems I Fund. Theory Appl., 47 (2000), 1089-1092. 1

[3] C. Briat, Convex conditions for robust stability analysis and stabilization of linear aperiodic impulsive and sampled-data systems under dwell-time constraints, Automatica, 49 (2013), 3449-3457. 3.2

[4] C. Briat, Dwell-time stability and stabilization conditions for linear positive impulsive and switched systems, Nonlinear Anal. Hybrid Syst., 24 (2017), 198-226. 1

[5] C. Briat, A. Seuret, A looped-functional approach for robust stability analysis of linear impulsive systems, Systems Control Lett., 61 (2012), 980-988. 3.2

[6] C. Briat, A. Seuret, Convex dwell-time characterizations for uncertain linear impulsive systems, IEEE Trans. Automatic Control, 57 (2012), 3241-3246. 3.2

[7] T. Chen, Global exponential stability of delayed Hopfield neural networks, Neural Netw., 14 (2001), 977-980. 1

[8] W.-H. Chen, Z. Ruan, W. X. Zheng, Stability and $\mathrm{l}_{2}$-gain analysis for impulsive delay systems: An impulse-timedependent discretized lyapunov functional method, Automatica J. IFAC, 86 (2017), 129-137. 3.5

[9] W.-H. Chen, W. X. Zheng, Global exponential stability of impulsive neural networks with variable delay: an LMI approach, IEEE Trans. Circuits Syst. I. Reg. Pap., 56 (2009), 1248-1259. 1

[10] S. R. Chu, R. Shoureshi, M. Tenorio, Neural networks for system identification, IEEE Control Syst. Mag., 10 (1990), 31-35. 1

[11] M. A. Davó, A. Baños, F. Gouaisbaut, S. Tarbouriechc, A. Seuret, Stability analysis of linear impulsive delay dynamical systems via looped-functionals, Automatica J. IFAC, 81 (2017), 107-114. 3.4, 3.5

[12] R. Goebel, R. G. Sanfelice, A. R. Teel, Hybrid dynamical systems. modeling, stability, and robustness, Princeton University Press, New Jersey, (2012). 1

[13] Z. Guo, J. Wang, Z. Yan, Attractivity analysis of memristor-based cellular neural networks with time-varying delays, IEEE Trans. Neural Netw. Learn. Syst., 25 (2014), 704-717. 1

[14] Y. He, M.-D. Ji, C.-K. Zhang, M. Wu, Global exponential stability of neural networks with time-varying delay based on free-matrix-based integral inequality, Neural Netw., 77 (2016), 80-86. 1

[15] W. He, F. Qian, J. Cao, Pinning-controlled synchronization of delayed neural networks with distributed-delay coupling via impulsive control, Neural Netw., 85 (2017), 1-9. 1

[16] M.-J. Hu, J.-W. Xiao, R.-B. Xiao, W.-H. Chen, Impulsive effects on the stability and stabilization of positive systems with delays, J. Franklin Inst., 354 (2017), 4034-4054. 1

[17] Y. Kao, C. Wang, L. Zhang, Delay-Dependent Robust Exponential Stability of Impulsive Markovian Jumping ReactionDiffusion Cohen-Grossberg Neural Networks, Neural Process. Lett., 38 (2013), 321-346. 1

[18] H. R. Karimi, H. Gao, New delay-dependent exponential $\mathrm{H}_{\infty}$ synchronization for uncertain neural networks with mixed time delays, IEEE Trans. Syst., Man, Cybern. B, Cybern., 40 (2010), 173-185. 1

[19] C. Li, S. Wu, G. G. Feng, X. Liao, Stabilizing Effects of Impulses in Discrete-Time Delayed Neural Networks, IEEE Trans. Neural Netw., 22 (2011), 323-329. 1

[20] X. Li, X. Zhang, S. Song, Effect of delayed impulses on input-to-state stability of nonlinear systems, Automatica J. IFAC, 76 (2017), 378-382. 1

[21] Y. Liu, Z. Wang, X. Liu, Global exponential stability of generalized recurrent neural networks with discrete and distributed delays, Neural Netw., 19 (2006), 667-675. 1

[22] J. Louisell, New examples of quenching in delay differential equations having time-varying delay, in Proceedings of the 4th European Control Conference, (1999). 3.6

[23] J. Lu, D. W. C. Ho, J. Cao, A unified synchronization criterion for impulsive dynamical networks, Automatica J. IFAC, 46 (2010), 1215-1221. 1, 4.1,1

[24] K. Mathiyalagan, H. Su, P. Shi, R. Sakthivel, Exponential $\mathrm{H}_{\infty}$ filtering for discrete-time switched neural networks with random delays, IEEE Trans. Cybern., 45 (2015), 676-687. 1

[25] S. R. Naidu, E. Zafiriou, T. J. McAvoy, Use of neural networks for sensor failure detection in a control system, IEEE Control Syst. Mag., 10 (1990), 49-55. 1

[26] A. Papachristodoulou, M. Peet, S. I. Niculescu, Stability analysis of linear systems with time-varying delays: Delay uncertainty and quenching, Proceedings of the 46th IEEE Conference on Decision and Control, New Orleans, LA, USA, 2007 (2007), 2117-2122. 3.6

[27] A. Seuret, C. Prieur, S. Tarbouriech, L. Zaccarian, Event-triggered control via reset control systems framework, IFACPapersOnLine, 49 (2016), 170-175. 3.4

[28] Q. Song, H. Yan, Z. Zhao, Y. Liu, Global exponential stability of impulsive complex-valued neural networks with both asynchronous time-varying and continuously distributed delays, Neural Netw., 81 (2016), 1-10. 1

[29] Y. Tang, H. Gao, J. Kurths, Multiobjective identification of controlling areas in neuronal networks, IEEE/ACM Trans. Comput. Biol. Bioinform., 10 (2013), 708-720. 1

[30] Y. Tang, H. Gao, J. Kurths, Robust $\mathrm{H}_{\infty}$ self-triggered control of networked systems under packet dropouts, IEEE Transactions on Cybernetics, 46 (2016), 3294-3305. 
[31] Y. Tang, H. Gao, J. Lu, J. Kurths, Pinning distributed synchronization of stochastic dynamical networks: a mixed optimization approach, IEEE Trans. Neural Netw. Learn. Syst., 25 (2014), 1804-1815.

[32] Y. Tang, Z. Wang, H. Gao, H. Qiao, J. Kurths, On controllability of neuronal networks with constraints on the average of control gains, IEEE Trans. Cybern., 44 (2014), 2670-2681. 1

[33] H. Wang, S. Duan, C. Li, L. Wang, T. Huang, Stability of impulsive delayed linear differential systems with delayed impulses, J. Franklin Inst., 352 (2015), 3044-3068. 3.5

[34] W. Wang, A. R. Teel, D. Nešić, Analysis for a class of singularly perturbed hybrid systems via averaging, Automatica J. IFAC, 48 (2012), 1057-1068. 1

[35] X. Wu, Y. Tang, W. Zhang, Input-to-state stability of impulsive stochastic delayed systems under linear assumptions, Automatica J. IFAC, 66 (2016), 195-204. 1, 3.3

[36] Y. Xia, C. Sun, W. X. Zheng, Discrete-time neural network for fast solving large linear $\mathrm{L}_{1}$ estimation problems and its application to image restoration, IEEE Trans. Neural Netw. Learn. Syst., 23 (2012), 812-820. 1

[37] X. Zhang, C. Li, T. Huang, Hybrid impulsive and switching Hopfield neural networks with state-dependent impulses, Neural Netw., 93 (2017), 176-184. 1

[38] W. Zhang, Y. Tang, J.-A. Fang, X. Wu, Stability of delayed neural networks with time-varying impulses, Neural Netw., 36 (2012), 59-63. 1, 3.3, 4.1

[39] W. Zhang, Y. Tang, Q. Miao, W. Du, Exponential synchronization of coupled switched neural networks with modedependent impulsive effects, IEEE Trans. Neural Netw. Learn. Syst., 24 (2013), 1316-1326. 1

[40] W. Zhang, Y. Tang, Q. Miao, J.-A. Fang, Synchronization of stochastic dynamical networks under impulsive control with time delays, IEEE Trans. Neural Netw. Learn. Syst., 25 (2014), 1758-1768. 1

[41] W. Zhang, Y. Tang, X. Wu, J.-A. Fang, Synchronization of nonlinear dynamical networks with heterogeneous impulses, IEEE Trans. Circuits Syst. I, Reg. Pap., 61 (2014), 1220-1228. 3

[42] X. Zhao, L. Zhang, P. Shi, M. Liu, Stability and stabilization of switched linear systems with mode-dependent average dwell time, IEEE Trans. Automat. Control, 57 (2012), 1809-1815. 1

[43] S. Zhu, M. Shen, C.-C. Lim, Robust input-to-state stability of neural networks with Markovian switching in presence of random disturbances or time delays, Neurocomputing, 249 (2017), 245-252. 1 